\section{LA-UR-}

Approved for public release: distribution is unlimited.

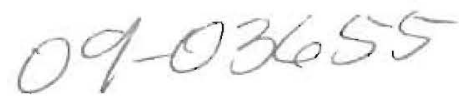

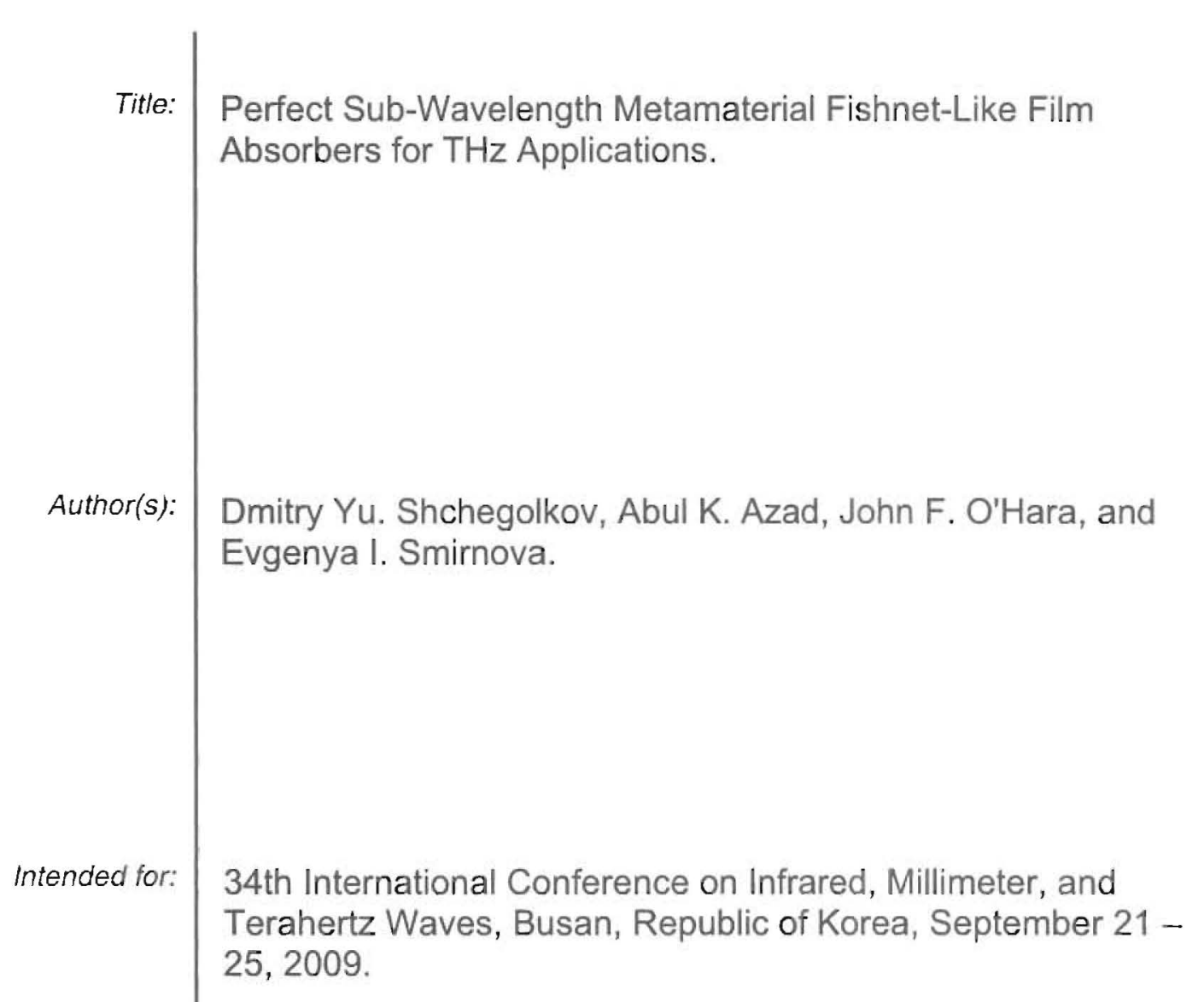

- Los Alamos

- EST.1943 -

\begin{abstract}
Los Alamos National Laboratory, an affirmative action/equal opportunity employer, is operated by the Los Alamos National Security, LLC for the National Nuclear Security Administration of the U.S. Department of Energy under contract DE-AC52-06NA25396. By acceptance of this article, the publisher recognizes that the U.S. Government retains a nonexclusive, royalty-free license to publish or reproduce the published form of this contribution, or to allow others to do so, for U.S. Government purposes. Los Alamos National Laboratory requests that the publisher identify this article as work performed under the auspices of the U.S. Department of Energy. Los Alamos National Laboratory strongly supports academic freedom and a researcher's right to pubtish; as an institution, however, the :-aboratory does not endorse the viewpoint of a publication or guarantee its technical correctness.
\end{abstract}




\title{
Perfect Sub-Wavelength Metamaterial Fishnet-Like Film Absorbers for THz Applications
}

\author{
Dmitry Yu. Shchegolkov, Abul K. Azad, John F. O'Hara, Evgenya I. Smirnova \\ Los Alamos National Laboratory, Los Alamos, NM 87545
}

\begin{abstract}
We present two designs of robust, easy to manufacture metamaterial-based films of sub-wavelength thickness capable of full absorption of the incident terahertz (THz) radiation at certain frequencies. Both designs can be either made polarization sensitive, or have $90^{\circ}$ rotation symmetry, which works equally well for waves of any polarization provided the incident angle is zero. All our designs work for a wide range of angles of incidence, and even if the films are optimized for normal incidence the absorption remains greater than $99 \%$ for angles up to $\sim 35^{\circ}$ in the TE and $\sim 65^{\circ}$ in the TM case. In the first design the maximum absorption frequency shifts considerably with angle, and in the second design the maximum absorption frequency remains almost the same at any angle. Theory, simulation data, and recent experimental results are all in a good agreement, and will be reported in the presentation. Having a low heat capacity these absorbers combined with thermo detectors can be utilized for precise frequency-selective detection of $\mathrm{THz}$ radiation.
\end{abstract}

\section{INTRODUCTION AND BACKGROUND}

$\mathrm{M}$ ETAMATERIALS are artificially-created media wi uniquely engineered electric permittivities and magnet permeabilities [1]. With metamaterials one can create resonant left-handed media with a negative index of refractic and negative phase velocity, and also a non-reflecting med with a wave impedance equal to that of the free space. Due the lack of naturally occurring materials for radiation contrc the $\mathrm{THz}$ frequency band is considered to be the one that ce greatly benefit from the metamaterial technology.

\section{RESULTS}

The last few years saw a blossom in development of differe kinds of metamaterials for $\mathrm{THz}$ frequencies, including tho: for absorber applications. There are already many know metamaterial film absorbers, including sub-wavelength fil absorbers, which were experimentally demonstrated to hat close to $100 \%$ wave absorption efficiency [2]. The ma drawback of those designs is that they employ resonators , rather complicated shape with many fine details and therefo are not easy to fabricate and are sensitive to distortions. $\mathrm{W}$ propose novel absorbers based on a simple resonator desig similar to the one that has already been successfully fabricats at optical frequencies: the fishnet structure (Fig. la) [3]. gold ground plate is used on the back side of the absorber to limit the absorber thickness and prevent any transmission. The front side of the absorber has a gold fishnet pattern and the space between these two layers of gold is filled with a lossy dielectric. In our case this dielectric was Kapton with loss tangent of 0.05 and permittivity of 3.4. The principle of operation is similar to a Fabry-Perot cavity with a semitransparent mirror and the ohmic loss equal to the radiation loss through this mirror. However our design has a subwavelength thickness.
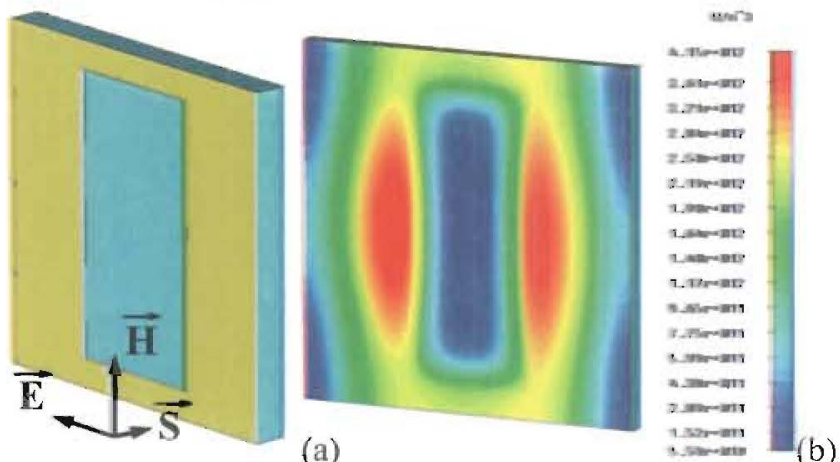

Fig. 1. The elementary cell of the metamaterial film: an absorbing Kapton layer is contained in between two layers of gold, one of which has a rectangular opening in it. The absorber consists of a $2 \mathrm{D}$ array of the above elementary cells (a). Losses in the center section of the film, proportional to the distribution of the electric field energy density (b)

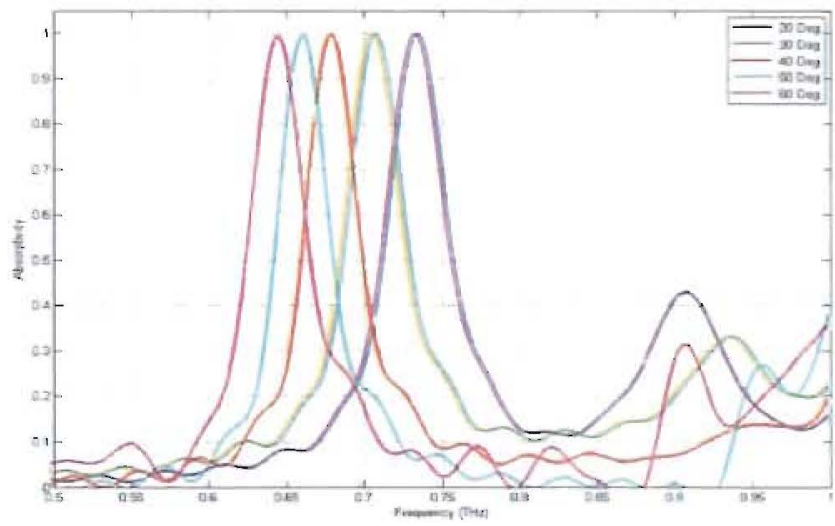

(a)

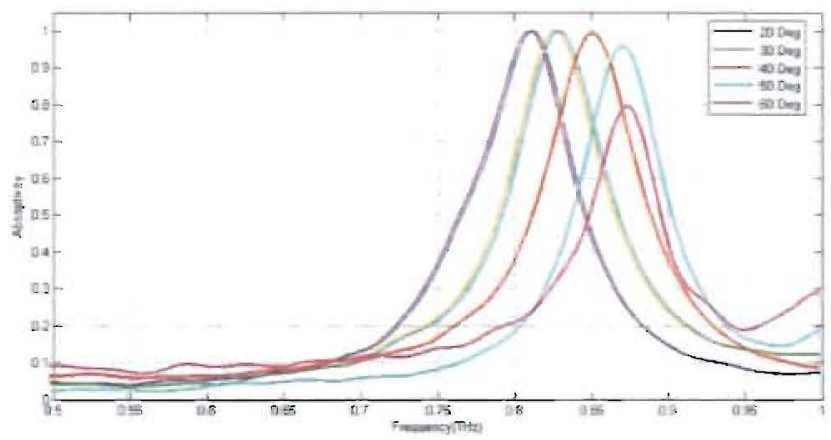

(b)

Fig. 2. Measured absorption for the TE (a) and the TM (b) incident waves for different angles of incidence. In the experiment the kapton layer thickness was $23 \mu \mathrm{m}$, gold layer thickness $0.3 \mu \mathrm{m}$, period of the structure $180 \mu \mathrm{m}$ and the size of the rectangular openings $150 \mu \mathrm{m} \times 75 \mu \mathrm{m}$. Maximum absorption frequency is red-shifted with angle for the TM case, and blue-shifted for the TE case.

The robustness of our design is determined by two factors. 
First, the absorber performance depends mostly on integral geometry characteristics of the structure. Second, the electromagnetic power is dissipated mostly in the dielectric and only a very small fraction is dissipated in the metal. This makes it less sensitive to the conducting properties of the metal surfaces, which are hard to measure and control. The simulated loss distribution inside the Kapton is shown in Fig. Ib. We did all the absorber simulations in CST Microwave Studio(B) $[4]$.

The fishnet-like absorber shown in Fig. la was tested experimentally. Our set-up allowed us to measure reflection in the range of incident angles in between 20 and 60 degrees and to use any of two primary linear polarizations. Due to a small period of the pattern, there could only be a specular reflection. Although the fabricated sample thickness of the Kapton layer came out to be $40 \%$ bigger than the optimum value derived from simulations, the absorber demonstrated close to the $100 \%$ absorption up to the very large angles of incidence, and it worked better for the TM incident waves than for waves incident in the other plane (Fig. 2). The absorber showed almost no absorption for the orthogonal polarization, having electric field component parallel to the longer sides of the rectangular opening.

Our second version of the absorber is based on a natural generalization of the fishnet structure pattern for the polarization insensitive case (Fig. 3a). Similar to the previous version (Fig. la), it can also be optimized to provide the full absorption at any chosen frequency and it also works for a wide range of incidence angles (Fig. 3b). The thickness of the Kapton layer corresponds to the one-way wave phase advance of only about 30 degrees.

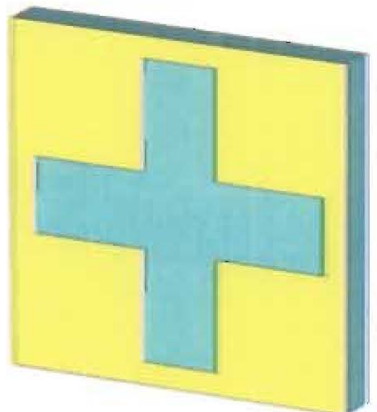

(a)

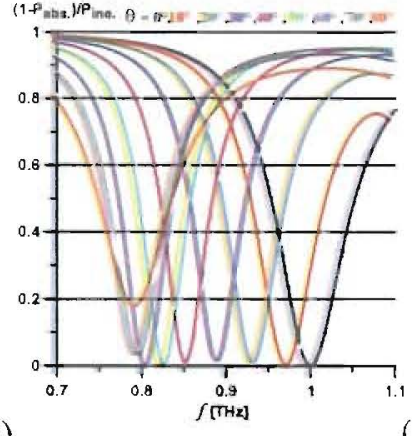

(b)

Fig. 3. The elementary cell of the polarization insensitive metamaterial film absorber (a). Corresponding reflected power as a function of frequency for different incident angles. $\theta$. for thc TM wave obtained from simulations (b). The perfect absorption takes place at both $0^{\circ}$ angle of incidence and at about $55^{\circ}$.

In both designs versions (Fig. 1a, 3a) the frequency corresponding to the absorption peak depends strongly on the angle of incidence (Fig. 2, 3b). This can benefit some applications such as the mechanically frequency tunable absorbers but can be a disadvantage for others. It is possible to eliminate this angle dependence almost completely by implementing a complementary pattern designs: noncontinuous stripes and crosses shown in Fig. 4. Note that the electromagnetic field orientation in Fig. $4 \mathrm{a}$ is different from the one in Fig. Ia. The absorber in Fig. 4 b resembles circuit analog absorbers in [5], which have one of the best bandwidth for given thickness, with the only difference that in our case the loss is not in the metallic cross, but in the dielectric.
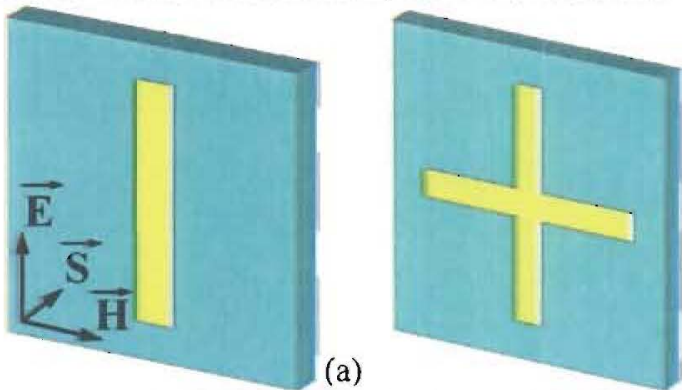

(b)

Fig. 4. Perfect absorbers of a complementary shape to those shown in Fig. la and $3 \mathrm{a}$. Periodic cell of the absorber film for a single linear polarization (a), and for an arbitrary polarization (b) of the incident waves.

Simulated absorber performance of the cross-patterned film for the angles of incidence up to 70 degrees is presented in Fig. 5. Maximum absorption frequency shifts almost negligibly with an angle and the shift has the opposite sign compared to the first set of absorbers.
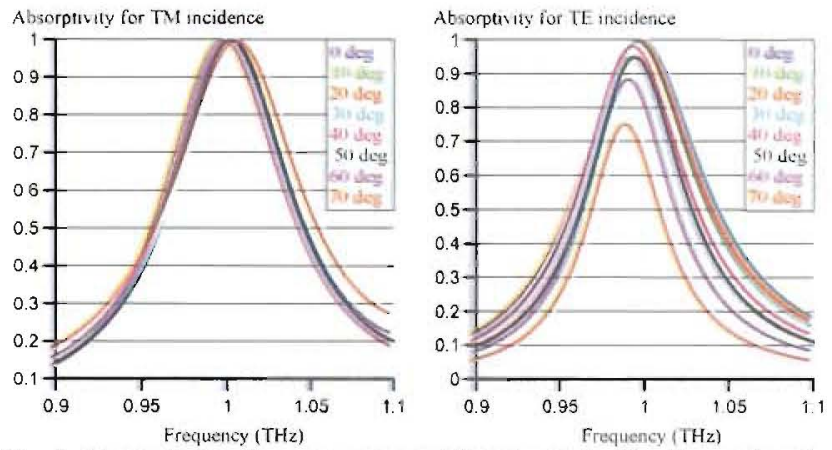

Fig. 5. Absorptivity of a cross-pattemed film shown in Fig. $4 b$ as a function of frequency for different incidence angles and two kinds of linear polarization.

It is worth noting, that the designs described above can be modified to dynamically control the maximum absorption frequency by implementing dielectrics with controlled permittivity or to dynamically control the value of the absorption peak by implementing semiconductors with controlled conductivity.

The metafilm absorbers may be utilized in $\mathrm{THz}$ detectors, nonreflecting surfaces, thermal imaging, and narrow band $\mathrm{THz}$ sources.

The authors gratefully acknowledge the support of the U.S. Department of Energy through the LANL/LDRD Program.

\section{REFERENCES}

[1] V.G. Veselago, Sov. Phys. Uspekhi. 10 (4), 509 (1968).

[2] H. Tao, C.M. Bingham, A.C. Strikwerda et al, Phys. Rev. B 78, $241103(\mathrm{R})(2008)$

[3] J. Valentine, S. Zhang, T. Zentgraf et al. doi:10,1038/nature07247 (2008).

[4] Microwave Studio, Computer Simulation Technology, www.cst.com.

[5] Ben A. Munk, Frequency selective surfaces: theory and design. John Wiley \& Sons (2000). 